\title{
A double inequality involving Erdős-Borwein constants
}

\author{
Mircea Merca
}




\section{A DOUBLE INEQUALITY INVOLVING ERDŐS-BORWEIN CONSTANTS}

\section{MIRCEA MERCA}

Received 20 May, 2014

Abstract. In this note, the author proves a double inequality by introducing an approximation of Erdôs-Borwein constants.

2010 Mathematics Subject Classification: 26D15; 26D07; 30B10

Keywords: inequalities, number of divisors, series

\section{INTRODUCTION}

Recently, after an experiment, we stumbled upon the following double inequality

$$
\left(x-\frac{1}{x+1}\right)^{-1}<\sum_{n=1}^{\infty} \frac{1}{1+x+x^{2}+\cdots+x^{n}}<\left(x-\frac{1}{x}\right)^{-1}, \quad x>1 .
$$

In this paper, we present a proof of this inequality using several tools from number theory.

Recall that a Mersenne number is a positive integer that is one less than a power of two:

$$
2^{n}-1
$$

The sum of the reciprocals of all Mersenne numbers, namely

$$
E=\sum_{n=1}^{\infty} \frac{1}{2^{n}-1}=1.6066951524152917637833 \ldots
$$

is known as the Erdős-Borwein constant. This mysterious number is known to be irrational, as shown by Erdős [4] in 1948.

More recently, using Padé approximant techniques, P. Borwein [2] established the irrationality of more general numbers

$$
E B(x)=\sum_{n=1}^{\infty} \frac{1}{x^{n}-1}
$$


for $x$ a positive integer, $x>1$. We remark that the numbers $E B(x)$ can be written as

$$
E B(x)=\frac{1}{x-1}+\frac{1}{x-1} \sum_{n=1}^{\infty} \frac{1}{1+x+x^{2}+\cdots+x^{n}}, \quad x>1 .
$$

We have the following approximation of these irrational numbers.

Theorem 1. Let $x$ be a real number such that $x>1$. Then

$$
\begin{aligned}
\frac{1}{x} & +\frac{1}{x^{4}} \cdot \frac{x^{2}+1}{x+1}<\sum_{n=1}^{\infty} \frac{1}{1+x+x^{2}+\cdots+x^{n}} \\
& <\frac{x}{x^{2}-1}-\frac{x}{x^{5}-1}+\frac{x}{x^{6}-1}-\frac{x}{x^{7}-1} .
\end{aligned}
$$

It is clear that this series has an asymptotic behaviour, i.e.,

$$
\sum_{n=1}^{\infty} \frac{1}{1+x+x^{2}+\cdots+x^{n}} \sim \frac{1}{x} \quad(x \rightarrow \infty) .
$$

The case $x=2$ of this theorem can be written as

In other words, we have

$$
\frac{29}{48}<E-1<\frac{153320}{248031} \text {. }
$$

$$
1.60416 \ldots<E<1.61814 \ldots
$$

\section{Proof of Theorem 1}

Let $n$ be a positive integer. The divisors function $\tau(n)$ is defined as the number of divisors of $n$, unity and $n$ itself included, i.e.,

$$
\tau(n)=\sum_{d \mid n} 1
$$

In number theory, the inequality

$$
\tau(n) \leqslant\lfloor 2 \sqrt{n}\rfloor
$$

is well-known. It is an easy exercise to show that

$$
\lfloor 2 \sqrt{n}\rfloor<1+\left\lfloor\frac{n}{2}\right\rfloor-\left\lfloor\frac{n}{5}\right\rfloor+\left\lfloor\frac{n}{6}\right\rfloor-\left\lfloor\frac{n}{7}\right\rfloor, \quad n \geqslant 36
$$

and

$$
\tau(n) \leqslant 1+\left\lfloor\frac{n}{2}\right\rfloor-\left\lfloor\frac{n}{5}\right\rfloor+\left\lfloor\frac{n}{6}\right\rfloor-\left\lfloor\frac{n}{7}\right\rfloor, \quad n<36 .
$$

Clearly,

$$
\tau(n) \leqslant 1+\left\lfloor\frac{n}{2}\right\rfloor-\left\lfloor\frac{n}{5}\right\rfloor+\left\lfloor\frac{n}{6}\right\rfloor-\left\lfloor\frac{n}{7}\right\rfloor,
$$

for any positive integer $n$. 
To prove the inequality we consider: the generating function of $\tau(n)[1$, s. 24.3.3],

$$
\sum_{n=1}^{\infty} \tau(n) q^{n}=\sum_{n=1}^{\infty} \frac{q^{n}}{1-q^{n}}, \quad|q|<1,
$$

the generating function of 1 ,

$$
\sum_{n=0}^{\infty} q^{n}=\frac{1}{1-q}, \quad|q|<1,
$$

and the generating function of $\lfloor n / k\rfloor$,

$$
\sum_{n=0}^{\infty}\left\lfloor\frac{n}{k}\right\rfloor q^{n}=\frac{q^{k}}{(1-q)\left(1-q^{k}\right)}, \quad|q|<1,
$$

where $k$ is a positive integer.

For $0<q<1$, we can write

$$
\begin{aligned}
\sum_{n=1}^{\infty} \frac{q^{n}}{1-q^{n}}<\sum_{n=1}^{\infty}(1 & \left.+\left\lfloor\frac{n}{2}\right\rfloor-\left\lfloor\frac{n}{5}\right\rfloor+\left\lfloor\frac{n}{6}\right\rfloor-\left\lfloor\frac{n}{7}\right\rfloor\right) q^{n} \\
=\frac{q}{1-q} & +\frac{q^{2}}{(1-q)\left(1-q^{2}\right)}-\frac{q^{5}}{(1-q)\left(1-q^{5}\right)} \\
& +\frac{q^{6}}{(1-q)\left(1-q^{6}\right)}-\frac{q^{7}}{(1-q)\left(1-q^{7}\right)}
\end{aligned}
$$

Thus we deduce that

or

$$
\begin{aligned}
\sum_{n=2}^{\infty} \frac{q^{n}}{1-q^{n}}< & \frac{q^{2}}{(1-q)\left(1-q^{2}\right)}-\frac{q^{5}}{(1-q)\left(1-q^{5}\right)} \\
& \quad+\frac{q^{6}}{(1-q)\left(1-q^{6}\right)}-\frac{q^{7}}{(1-q)\left(1-q^{7}\right)}, \quad 0<q<1
\end{aligned}
$$

$$
\sum_{n=1}^{\infty} \frac{q^{n}}{1+q+q^{2}+\cdots+q^{n}}<\frac{q}{1-q^{2}}-\frac{q^{4}}{1-q^{5}}+\frac{q^{5}}{1-q^{6}}-\frac{q^{6}}{1-q^{7}}, \quad 0<q<1 .
$$

Replacing $q$ by $1 / x$ in this inequality, we get

$$
\sum_{n=1}^{\infty} \frac{1}{1+x+x^{2}+\cdots+x^{n}}<\frac{x}{x^{2}-1}-\frac{x}{x^{5}-1}+\frac{x}{x^{6}-1}-\frac{x}{x^{7}-1}, \quad x>1 .
$$

On the other hand, due to Clausen's [3], we have the following identity:

$$
\sum_{n=1}^{\infty} \frac{q^{n}}{1-q^{n}}=\sum_{n=1}^{\infty} q^{n^{2}} \frac{1+q^{n}}{1-q^{n}}, \quad|q|<1
$$


For $x>1$, we obtain

$$
\begin{aligned}
\sum_{n=1}^{\infty} \frac{1}{1+x+x^{2}+\cdots+x^{n}} & =(x-1) E B(x)-1 \\
& =(x-1) \sum_{n=1}^{\infty} x^{-n^{2}} \frac{x^{n}+1}{x^{n}-1}-1 \\
& >(x-1)\left(\frac{1}{x} \cdot \frac{x+1}{x-1}+\frac{1}{x^{4}} \cdot \frac{x^{2}+1}{x^{2}-1}\right)-1 \\
& =\frac{1}{x}+\frac{1}{x^{4}} \cdot \frac{x^{2}+1}{x+1}
\end{aligned}
$$

and Theorem 1 is proved.

\section{CONCLUding REMARKS}

Lower and upper bounds of Erdős-Borwein constants has been introduced in the paper. For $x>1$, it is an easy exercise to prove that

$$
\frac{x+1}{x^{2}+x-1}<\frac{1}{x}+\frac{1}{x^{4}} \cdot \frac{x^{2}+1}{x+1}
$$

and

$$
\frac{x}{x^{2}-1}-\frac{x}{x^{5}-1}+\frac{x}{x^{6}-1}-\frac{x}{x^{7}-1}<\frac{x}{x^{2}-1} \text {. }
$$

So by Theorem 1, we derive the double inequality (1.1) that can be written in terms of Erdős-Borwein constants as follows

Corollary 1. For $x>1$,

$$
\left(x-\frac{1}{x+1}\right)^{-1}<(x-1) E B(x)-1<\left(x-\frac{1}{x}\right)^{-1} \text {. }
$$

\section{ACKNOWLEDGEMENT}

Special thanks go to the anonymous referees for their helpful assistance. The author expresses his gratitude to Dr. Oana Merca for the careful reading of the manuscript and helpful remarks.

\section{REFERENCES}

[1] M. Abramowitz and I. A. Stegun, Handbook of Mathematical Functions with Formulas, Graphs, and Mathematical Tables. New-York: Dover Publications, 1972.

[2] P. Borwein, "On the irrationality of certain series," Math. Proc. Cambridge Philos. Soc., vol. 112, pp. 141-146, 1992.

[3] T. Clausen, "Beitrag zur theorie der reihen," Journal für die reine und angewandte Mathematik, vol. 3, pp. 92-95, 1828. 
[4] P. Erdős, "On arithmetical properties of lambert series," J. Indian Math. Soc., vol. 12, pp. 63-66, 1948.

Author's address

Mircea Merca

University of Craiova, Department of Mathematics, 200585 Craiova, Romania

Current address: Nicolae Grigorescu National College, Department of Informatics, 105600 Câmpina, Romania

E-mail address: mircea.mercaeprofinfo.edu.ro 